\title{
Tasa de mortalidad y años potenciales de vida perdidos por suicidio en comunas y barrios de Medellín, Colombia, 2010 y 2012
}

\author{
Angie Vanessa Velásquez Vélez ${ }^{1}$, Grisales Hugo ${ }^{2}$ hugo.grisales@udea.edu.co \\ 1 Estudiante de Gerencia de Sistemas de Información en Salud, Joven investigadora del Grupo de Demografía y Salud, Facultad Nacional de Salud Pública, \\ Universidad de Antioquia. \\ 2 Doctor en Epidemiología, Profesor Titular de la Facultad Nacional de Salud Pública, Universidad de Antioquia,
}

Fecha de recepción marzo 14 de 2016 Fecha de correcciones agosto 6 de 2016 Fecha de aceptación septiembre 30 de 2016 Fecha de publicación enero 31 de 2017

\begin{abstract}
Resumen
Introducción: El suicidio es un problema de salud pública por el impacto negativo que ha tenido sobre los colectivos. Se calcula la tasa de mortalidad y los años potenciales de vida perdidos (APVP) por suicidio además de la distribución geográfica. Materiales y métodos: Estudio descriptivo, con información secundaria facilitada por el Departamento Nacional de Estadística (DANE); se calcularon las tasas de mortalidad y de los APVP por suicidio, tomando como referente la esperanza de vida al nacer de Medellín para los años 2010 y 2012, además de la construcción de la distribución geográfica del suicidio por comuna y barrio de la ciudad de Medellín. Resultados: En Medellín, para los dos años de estudio, se presentaron 253 suicidios, de los cuales el 81,4\% fueron hombres. Las tasas de mortalidad para 2010 y 2012 fueron de 5,2 y 5,4 por cada cien mil habitantes, respectivamente. Las personas de los grupos de edad de 20 a 29 años, seguido del de 10 a 19 años fueron los que mayor APVP aportaron. Se destacó el hecho de que las comunas La Candelaria y El Poblado contribuyeron en mayor magnitud con los APVP en ambos sexos y en los barrios Calle Nueva, Guayaquil, Pablo VI y Caribe y Cerro Nutibara se presentó situación ídem. Discusión: Aunque el suicidio es un evento probablemente evitable, aún tiene gran incidencia especialmente en los hombres en edad productiva., lo que sugiere intervenciones en salud mental que apunten a la disminución de tal flagelo.
\end{abstract}

Palabras clave: Suicidio, Tasas de Mortalidad, APVP, Medellín, Distribución Gráfica.

Copyright (C) Facultad de Ciencias de la Salud de la Universidad Tecnológica de Pereira. 1995-2017. Todos los derechos reservados *
Mortality and potential years of life lost by suicide by Commune and neighborhoods of Medellin, Colombia, 2010 and 2012

\section{Abstract}

Introduction: Suicide is a public health problem because of the negative impact it has had on the collectives. The mortality rate and the years of potential life lost (YPLL) by suicide are estimated in addition to their geographical distribution. Materials and methods: Descriptive study, with secondary information provided by the National Department of Statistics (DANE); the mortality rates and the YPLL by suicide were calculated, taking as a reference the life expectancy at birth of Medellin for the years 2010 and 2012, in addition to the construction of the geographical distribution of suicide by commune and neighborhood of the city of Medellin. Results: In Medellin, for the two years of study, there were 253 suicides, $81.4 \%$ of which were men. The mortality rates for 2010 and 2012 were 5.2 and 5.4 per hundred thousand inhabitants, respectively. People in the age groups from 20 to 29 years, followed by the 10 to 19 years were the ones with the greatest contribution of YPLL. It was noted that the communes La Candelaria and El Poblado contributed to a greater extent with the YPLL in both genders, and in the suburbs Calle Nueva, Guayaquil, Pablo VI, Caribe and Cerro Nutibara a similar situation was found. Discussion: Although suicide is an event possibly avoidable, it still has a large impact especially in men in productive age, which suggests the need of future that mental health interventions in order to decrease this scourge.

Keywords: Suicide, Mortality Rates, YPLL, Medellin, Geographical Distribution.

Copyright $\odot$ Facultad de Ciencias de la Salud de la Universidad Tecnológica de Pereira. 1995-2017. All rights reserved ${ }^{*}$.

\section{Introducción}

En las últimas décadas el suicidio se ha convertido en un problema de salud pública por el impacto negativo que ha tenido sobre los colectivos, especialmente en países desarrollados, en particular cada 40 segundos una persona que intenta suicidarse, logra su objetivo, la muerte [1].

La Organización Mundial de la Salud -OMS- expresa que el problema del suicidio va más allá de las estadísticas, ya que en ocasiones los familiares lo ocultan, para evitar la estigmatización de la persona que ha tomado tal decisión [2].

Los factores que surgen del suicidio se asocian a problemáticas educativas y mentales, los que tienen un efecto sinérgico sobre la dinámica familiar, cargándoles consigo secuelas psicológicas, donde queda sensación de pérdida, legado de vergüenza, miedo, rechazo, enojo y culpa, aunado a sentimientos de tristeza, a síntomas físicos, hipersensibilidad, trastornos del apetito y del sueño [3]. De otro lado, los problemas mentales podrían desencadenar en el suicidio, como lo revela el hecho de que el $90 \%$ de las muertes, por causa del suicidio están asociadas a personas que sufren alguna enfermedad mental, hayan sido diagnosticadas o no con anterioridad [3]. La violencia es otro factor que puede contribuir a la explicación de tal evento. De acuerdo con la OMS, las proyecciones indican que para el año 2020 la depresión pasará a ser el problema número uno del mundo y causará el 3,4\% de la carga global de enfermedad. Se espera que con el incremento de la depresión durante este periodo, los suicidios aumenten significativamente, de 593.000 en 1990 a 995.000 en el año 2020 [4].

Diversos estudios demostraron que esta problemática ha tenido, históricamente, protagonismo no solo a nivel mundial sino a nivel nacional, En Colombia hacia el año 1999 el suicidio ya era considerado como la cuarta causa de muerte violenta, siendo los años 2000-2002 los que presentaron las tasas más altas de suicidio [5], aunque desde 1998 venía en ascenso la magnitud del problema. En términos de carga de la enfermedad, el suicidio representó 2,2\% de los APVP en Colombia en 2002, con especial afectación a la población joven. Este aumento fue más notable en las mujeres de 10 a 19 años y en los hombres de 20 a 29 años [6], lo que tiene repercusiones a nivel demográfico y laboral por tratarse de grupos etarios que están en proceso de desarrollo de su capacidad productiva y reproductiva.

Estudios recientes en Colombia, demostraron que hacia el año 2012 la tasa de suicidio fue de 4,1 suicidios por cada 100.000 habitantes con la misma tendencia que en el pasado respecto al sexo (son los hombres en mayor medida quienes mueren por suicidio) [7]; el grupo más afectado era aquel en el que se encontraban individuos entre 20-24 años con 16,3\% ${ }^{7}$; este panorama fue similar por Departamento donde las mayores tasas por 100.000 habitantes se registraron en Arauca (8,3), Risaralda (7,6), Casanare (7,7) Quindío (7,0), Putumayo $(6,3)$ y Antioquia (5,3) [8], y a nivel local en este último departamento, es la ciudad de Medellín la que ha presentado un importante aumento en las tasas de mortalidad por causas externas 
en general y en particular por el suicidio donde la tasa promedio en 2010 fue de 5,2 por cada cien mil habitantes en el que 50\% de los casos correspondían a personas de 30 años o menos [9]. Alrededor del evento indicado, el uso de agentes tóxicos, de proyectil de arma de fuego y armas corto punzantes eran los métodos más utilizados; se destacó además un perfil en donde los suicidas eran preferiblemente solteros, con antecedentes previos de intentos de suicidio [5] y con adicción al consumo de sustancias psicoactivas [7].

Para evitar la frecuencia con la que ocurre el suicidio la OMS ha presentado planes de prevención en el que se destaca especialmente el Plan Decenal de Salud Pública de Colombia 2012-2021, dentro de la dimensión convivencia social y salud mental se apunta al fomento del auto cuidado, a cambiar los imaginarios sociales de la salud mental y a la promoción de sus factores protectores en personas y poblaciones en riesgo, donde su meta a 2021 no solo es la disminución de la mortalidad por suicidio, sino las lesiones auto infligidas a 4.7 por 100.000 habitantes $[4,6]$; el propósito anterior refleja la necesidad de investigaciones locales con mayor detalle, por lo que en este estudio se describe la mortalidad y la magnitud de los años de vida potenciales perdidos por suicidio junto con la distribución gráfica por comuna y barrio de Medellín, Colombia entre los años 2010 y 2012, con el fin de evidenciar el impacto y magnitud del problema del suicidio, específicamente con indicadores que cuantifiquen las pérdidas en años que sufre esta población, lo que permitirá sustentar el proceso de la toma de decisiones o focalización respecto a la dirección de los recursos disponibles en promoción y prevención, que los hallazgos soporten la disminución progresiva del evento suicidio, como suceso probablemente evitable.

\section{Materiales y métodos}

Se trata de un estudio descriptivo con información proveniente de fuentes secundarias. La población de estudio fueron los registros de las defunciones tabulados por el Departamento Nacional de Estadística DANE, a nivel del micro dato en los años 2010 y 2012, dado que al momento del estudio se tenía la información oficial para el último año referido anteriormente. Un proceso de depuración de la información facilitó la captura de la comuna y del barrio de residencia del occiso lo que permitió una aproximación geográfica al comportamiento del evento de interés, por las diferentes comunas y barrios del Municipio de Medellín, Colombia de los años 2010 y 2012, y que están codificadas en la décima versión de la Clasificación Internacional de Enfermedades (CIE-10).

Se calcularon las distribuciones de frecuencias, los APVP y las tasas, de los años potenciales de vida perdidos por muerte prematura debido al suicidio (para ello se tomó en cuenta la esperanza de vida al nacer de Medellín para los años 2006-2010 la cual era de 77,6 años en mujeres y 72,8 en hombres y para 2011-2015 de 78,5 en mujeres y 75,5 en ese orden y la distribución espacial correspondiente a las distintas comunas y barrios de Medellín, Colombia, tomando como referencia la clasificación geográfico a nivel de comuna y barrio que hace Planeación Metropolitana de la ciudad de Medellín.

Se controló el sesgo de selección, eligiendo solo aquellas causas básicas de defunción que tuviesen asignado el código correspondiente según la CIE-10: (X60-X84) y que refiere el evento suicidio. Se excluyeron aquellos registros que no presentaron información de lugar de ocurrencia, para el caso de la representación gráfica en mapas de los APVP por suicidio.

El estudio se rigió bajo las consideraciones éticas de la Resolución No. 008430 de 1993 [10].

Los indicadores y representaciones pictóricas se obtuvieron mediante los oficios de los programas SPSS, versión $21^{\circledR}$, Excel y Arcgis, Arcmap, v $10^{\circ}$.

\section{Resultados}

Panorama general de la mortalidad por suicidio

En Medellín para los dos años de estudio se presentaron 253 suicidios, de los cuales el 81,4\% fueron hombres. En el año 2012 hubo un incremento del $4 \%$ en las defunciones respecto al año 2010 (48\%); cuando se estimaron las tasas de mortalidad para ambos años las diferencias no fueron marcadas, 5,2 y 5,4 por cien mil habitantes habitantes, para los años 2010 y 2012 respectivamente. Se destacó que las tasas de mortalidad más elevadas eran en hombres, sin embargo para ambos años no se presentaron diferencias importantes en la tasa de mortalidad por sexo aunque en el año 2012 hubo un leve incremento en dicho indicador en las mujeres, en una magnitud aproximada del $14 \%$.

Se destacó que por cada 100.000 hombres suicidas, aproximadamente 6 de ellos eran solteros al momento de la muerte, contrario a las mujeres que por cada 100.000, 1 de ellas eran casadas. Respecto a la causa básica de defunción se evidenció que el ahorcamiento fue el común denominador en ambos sexos, seguido en hombres por lesiones auto infligidas intencionalmente por disparo de otras armas de fuego y las no especificadas (17,5\%); y en mujeres por el hecho de saltar desde un lugar elevado (23\%).

Mortalidad general por sexo y grupos de edad.

En Medellín para los dos años de estudio la edad promedio de las personas que fallecieron a causa del suicidio fue de aproximadamente 33,5 años $(\mathrm{DE}=15,5)$, el $50 \%$ de las muertes se dio a los 29 años o menos; la edad mínima de ocurrencia de la misma fue de 10 años y la máxima a los 98 años. (Tabla 1).

Tabla 1. Distribución de frecuencias de las defunciones y tasas de mortalidad por suicidio por grupos etarios. Medellín, 2010 y 2012.

\begin{tabular}{|c|c|c|c|c|c|c|c|}
\hline \multirow{3}{*}{ Año de defunción } & \multicolumn{7}{|c|}{ Sexo } \\
\hline & \multirow{2}{*}{ Grupo de edad } & \multicolumn{2}{|c|}{ Hombres } & \multicolumn{2}{|c|}{ Mujeres } & \multicolumn{2}{|c|}{ Total } \\
\hline & & Muertes (\%) & Tasa* & Muertes $(\%)$ & Tasa* & Muertes $(\%)$ & Tasa* $^{*}$ \\
\hline \multirow{8}{*}{2010} & $<10$ años & $0(0,0)$ & 0,0 & $0(0,0)$ & 0,0 & $0(0,0)$ & 0,0 \\
\hline & 10-19 años & $17(16,5)$ & 17,6 & $4(20)$ & 4,3 & $21(17,1)$ & 5,9 \\
\hline & $20-29$ años & $36(34,9)$ & 37,7 & $6(30)$ & 6,1 & $42(34,1)$ & 10,8 \\
\hline & 30-39 años & $17(16,5)$ & 23,2 & $2(10)$ & 2,3 & $19(15,4)$ & 6,2 \\
\hline & 40-49 años & $13(12,6)$ & 15,2 & $5(25)$ & 4,7 & $18(14,6)$ & 4,8 \\
\hline & $50-59$ años & $12(11,6)$ & 16,6 & $3(15)$ & 3,4 & $15(12,2)$ & 4,9 \\
\hline & $60 \mathrm{y}+$ años & $8 \quad(7,8)$ & 32,3 & $0(0,0)$ & 0,0 & $8(6,5)$ & 5,5 \\
\hline & Total & $103(83,7)$ & 9,3 & $20(16,3)$ & 1,6 & $123(100)$ & 5,2 \\
\hline \multirow{8}{*}{2012} & $<10$ años & $0(0,0)$ & 0,0 & $0(0,0)$ & 0,0 & $0 \quad(0,0)$ & 0,0 \\
\hline & 10-19 años & $18(17,5)$ & 19,5 & $8(29,6)$ & 9,2 & $26(20,0)$ & 7,5 \\
\hline & $20-29$ años & $34(33,0)$ & 34,1 & $8(29,6)$ & 8,0 & $42(32,3)$ & 10,5 \\
\hline & 30-39 años & $20(19,4)$ & 26,6 & $3(11,1)$ & 3,4 & $23(17,7)$ & 7,2 \\
\hline & $40-49$ años & $14(13,6)$ & 17,0 & $4(14,8)$ & 4,0 & $18(13,8)$ & 4,9 \\
\hline & $50-59$ años & $8(7,8)$ & 10,5 & $3(11,1)$ & 3,2 & $11 \quad(8,5)$ & 3,3 \\
\hline & $60 \mathrm{y}+$ años & $9(8,7)$ & 31,1 & $1(3,7)$ & 1,6 & $10 \quad(7,7)$ & 6,3 \\
\hline & Total & $103(79,2)$ & 9,4 & $27(20,8)$ & 2,2 & $130(100,0)$ & 5,6 \\
\hline
\end{tabular}

* por cada 100.000 habitantes

Tabla 2. Indicador promedio de Tasas de APVP según sexo y grupo de edad, para los dos años de estudio, Medellín.

\begin{tabular}{|c|c|c|c|c|c|}
\hline \multirow[b]{2}{*}{ Edad } & \multicolumn{2}{|c|}{ Hombres } & \multicolumn{2}{|c|}{ Mujeres } & \multirow{2}{*}{$\begin{array}{c}\text { Total } \\
\text { Tasa total } \\
\end{array}$} \\
\hline & APVP (\%) & Tasa * & APVP (\%) & Tasa * & \\
\hline$<10$ años & $0,0 \quad(0,0)$ & 0,0 & $0,0 \quad(0,0)$ & 0,0 & 0,0 \\
\hline 10-19 años & $2007,1(24,1)$ & 1119,4 & $742,6(34,1)$ & 430,0 & 781,2 \\
\hline 20-29 años & $3480,8(41,8)$ & 1778,2 & $748,8 \quad(34,3)$ & 377,3 & 1073,0 \\
\hline 30-39 años & $1515,1(18,2)$ & 1044,0 & $223,7(10,3)$ & 132,5 & 553,9 \\
\hline $40-49$ años & $802,8 \quad(9,6)$ & 476,2 & $301,1 \quad(13,8)$ & 148,4 & 297,2 \\
\hline 50-59 años & $422,9 \quad(5,1)$ & 301,1 & $148,4 \quad(6,8)$ & 84,0 & 180,2 \\
\hline 60 y mas años & $97,6 \quad(1,2)$ & 72,9 & $15,2 \quad(0,7)$ & 8,0 & 35,1 \\
\hline Total & $8326,3(79,2)$ & 746,8 & $2179,8(20,7)$ & 173,9 & 443,7 \\
\hline
\end{tabular}

Se observó que para ambos años en estudio, las tasas de mortalidad por suicidio se presentaron, preferiblemente, entre 20 y 29 años; a partir de esta última se presentó una disminución marcada exceptuando para 2012 en el grupo de edad de 60 y más años que mostró un aumento en su tasa, la que fue equivalente a 6,3 defunciones por cada 100.000 habitantes. Según el sexo para el año 2010 tanto en hombres como en mujeres la mayor tasa se generó en el grupo de edad de 20 a 
29 años, seguido del grupo de edad de 60 y más años y 40 a 49 años, respectivamente; en el año 2012 se presentó similar comportamiento respecto a los hombres, aunque en las mujeres fue mayor entre 10 y 19 años (9,2 por cien mil habitantes) (Tabla 2).

Mortalidad general por lugar de ocurrencia del hecho y sexo. El $13,6 \%$ de las defunciones ocurrieron en la comuna $10 \mathrm{La}$ Candelaria, de acuerdo a la tasa de mortalidad, por cada 100.000 habitantes aproximadamente 36,4 se suicidaron, en segundo lugar se ubicó la comuna 14 El Poblado: allí por cada 100.000 habitantes ocurrieron aproximadamente 15 muertes a causa del suicidio, le siguieron las comunas Manrique, Villa Hermosa, Buenos Aires y Robledo en ese orden.

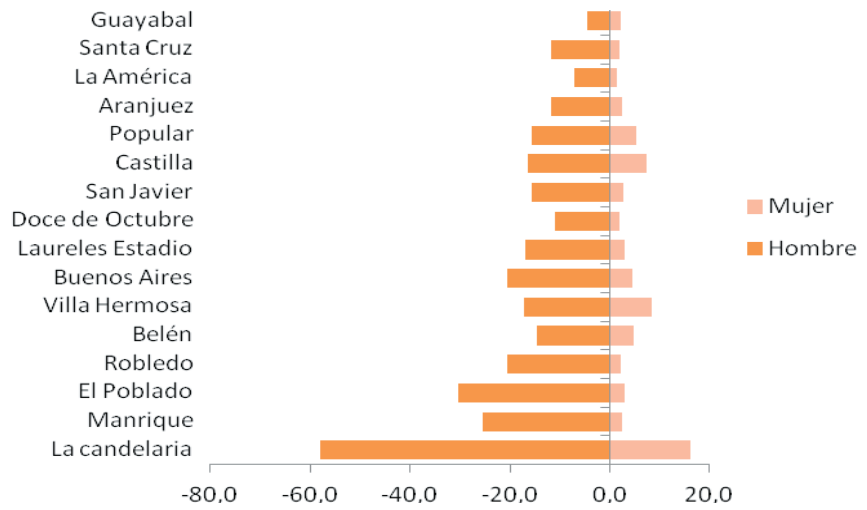

Figura 1. Tasas de mortalidad por Suicidio según sexo y comuna de Medellín, 2010 y 2012.

En la comuna donde se presentó mayor número de defunciones de hombres fue en la Candelaria, seguido de la comuna 14 El Poblado; la Comuna la Candelaria también fue donde se presentaron mayor número de defunciones en mujeres, seguidas de las presentadas en la Comuna 10 correspondiente a Villa Hermosa (Figura 1).

El barrio con mayor presentación de la mortalidad por suicidio fue La Candelaria, allí por cada 100.000 habitantes se presentaron aproximadamente 184 defunciones por suicidio, seguido de la Facultad de Minas, ubicado en la comuna 7 Robledo (164,3 por cien mil habitantes); las menores tasas de mortalidad por suicidio se generaron en los barrios Cristo rey y Moravia (5,0 y 5,1 por cien mil habitantes respectivamente). Se destacó de acuerdo al sexo que en el barrio Alejandría después de La Candelaria se generó la mayor tasa de mortalidad por suicidio en hombres, contrario a su tasa en las mujeres ya que esta era equivalente a cero. En los hombres la tercer tasa más elevada se presentó en San Javier No.1 (97,8 por cien mil habitantes) y en las mujeres el barrio con tasa más elevada fue la Facultad de Minas, seguido de La Candelaria.

\section{APVP por suicidio por año y sexo.}

En general para los dos años de estudio, se presentaron por cada 100.000 personas 460,1 APVP por suicidio, siendo el año 2012 el de mayor Tasa de APVP (482,9 APVP), es decir que la tendencia de 2010 a 2012 fue ascendente. Para ambos años, los hombres predominaron respecto a la pérdida de años potenciales de vida: por cada 100.000 hombres de Medellín en los años 2010 y 2012 se perdieron aproximadamente 373,3 años potenciales de vida. Las tasas de APVP según sexo, de acuerdo al año de defunción; para 2012 por cada 100.000 habitantes aumentó considerablemente en mujeres, pasando de 72,2 años a 101,4 APVP; en los hombres también se presentó un aumento (de 16 APVP), pero no tan relevante.

Tasa de APVP según comuna, barrio, sexo y su distribución espacial.

Tomando como referencia el lugar con mayor ocurrencia del hecho en el año 2010, se evidenció que fue la Comuna 10, La candelaria quien presentó la tasa total de APVP por suicidio más elevada (91,2 por cien mil habitantes respectivamente; correspondiéndole un 25,8\%), seguido de la comuna 3 , Manrique, la comuna 5, Castilla y la Comuna 1, Popular. Fue en la comuna 12 La América en donde se generó por cada 10.000 habitantes la menor tasa de mortalidad por suicidio, equivalente a 0,9 por diez mil habitantes respectivamente.

Según sexo, tanto en hombres como en mujeres la comuna 10 la Candelaria continuó el mismo patrón presentado en la tasa total de APVP, la comuna 12 La América siguió la de menor tasa.

En 2010 los barrios pertenecientes a la comuna 10, La Candelaria, que geográficamente están más cerca al rio Medellín fueron quienes aportaron mayor tasa de APVP por suicidio, tales como Calle Nueva a quien se le atribuyó el 51,2\% del total de APVP por suicidio de los barrios de Medellín, seguidamente se encontró el barrio Guayaquil; los barrios de la comuna 1 y la 5 también tuvieron altas tasas, como: Pablo VI y Caribe.

En el año 2012 el barrio Carlos E. Restrepo perteneciente a la comuna 11, Laureles-Estadio y el barrio La Candelaria de la comuna 10, La Candelaria fue donde se obtuvo las mayores tasas de APVP por suicidio (65,6 por mil habitantes) equivalente al 16,3\% de los APVP, y 36,11 por mil habitantes respectivamente También en el barrio Cerro Nutibara se generó una alta tasa de APVP, equivalente a 34,0 por cada 1.000 habitantes.

APVP por suicidio según año, sexo, grupo de edad: distribución geográfica espacial.

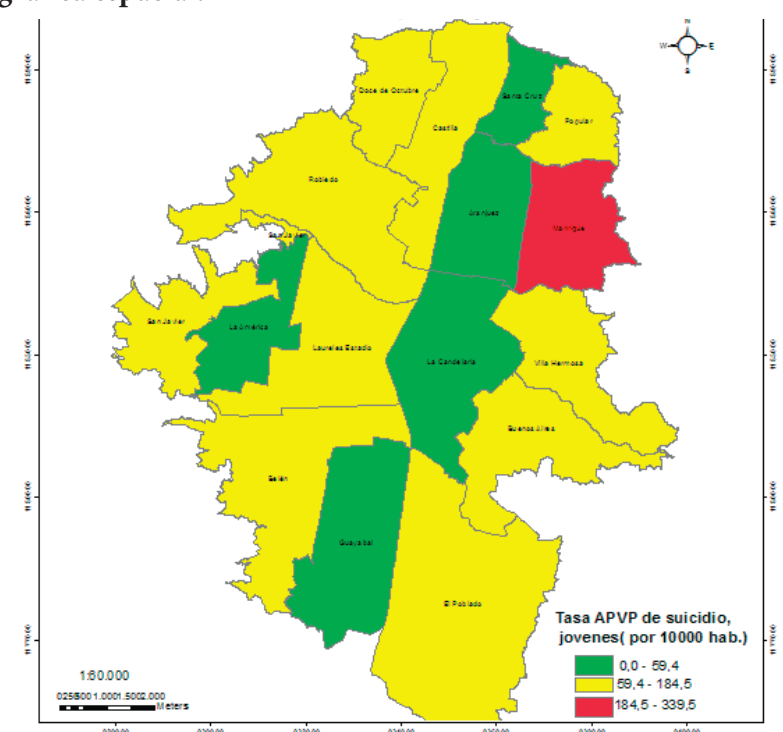

Figura 2. Tasa de APVP por suicidio en jóvenes, según comuna de ocurrencia del hecho, Medellín 2010 y 2012.

Los APVP más prevalentes por cada 100.000 hombres se generaron en ambos años de estudio en el grupo de edad de 20 a 29 años, seguido del grupo de edad de 10 a 19 años; en contraste, en las mujeres la pérdida de años por muerte prematura se concentró principalmente en el grupo de edad de 10 a 19 años, seguido del de edades entre 20 y 29 años (Ver tabla 2). Las tasas más elevadas en el grupo etario de 10 a 19 se presentaron en la Comuna 3 Manrique (272,8 por diez mil habitantes) y la 1, Popular (116 por mil habitantes). En 2012 las mayores tasas de suicidio se concentraron en la comuna 13 San Javier (110,7 por diez mil habitantes) y Doce de Octubre (91,7\%) (Ver Figura 2). Cuando se consideró la distribución del suicidio en la población de 20 a 59 años, las mayores tasas de APVP en el 2010 se presentaron en la comuna 10 La Candelaria y la 16, Belén, mientras que en el 2012 fue la comuna Manrique (272 por diez mil habitantes) quien presentó las tasas más elevadas de APVP por suicidio en ídem grupo de edad. Por su parte en la población de 60 y más años que murieron por el evento en cuestión, este ocurrió en mayor magnitud, en el año 2010, 
en las comunas de Belén (75 por diez mil habitantes), Guayabal (73 por diez mil habitantes) y La América (61por diez mil habitantes), en contraste con los indicadores para los jóvenes quienes en el periodo de tiempo estipulado, fueron quienes tuvieron menor aporte al indicador trazador en estas comunas. En 2012 también presento la mayor tasa de APVP por suicidio la Comuna 16 Belén, seguido de la comuna 10 La Candelaria.

\section{Discusión}

Los estudios de mortalidad tienen especial relevancia porque aportan información sobre las principales causas por las que muere la población lo que, en salud pública, es de vital importancia ya que sustenta las estrategias de intervención con enfoque científico; también los estudios de mortalidad aportan mejor comprensión de la dinámica de las diferentes causas de muerte y al tratamiento que a ellas se les debe generar, lo que por ende provoca la formulación de políticas públicas en pro de la prevención de ellas y en asegurar una mejor calidad de vida a la población. Para conocer el comportamiento de la mortalidad en una población existen indicadores clásicos y no clásicos; de los primeros están las tasas de mortalidad bruta y específica, entre la segunda los Años Potenciales de Vida Perdidos, APVP, usual en la medición de una componente de la causa de la enfermedad. Los APVP permiten evidenciar aspectos correspondientes a la medida del nivel y al cambio de la mortalidad en la población, es decir permite sustentar históricamente la mortalidad por causas de muerte, además de medir en forma aceptable el cambio de la mortalidad aunque aparentemente estos sean relativamente bajos [11].

En los últimos tiempos el suicidio, es considerado a nivel mundial la segunda causa principal de defunción en el grupo etario de 15 a 29 años, y en Colombia se ha posicionado como la cuarta causa de muerte violenta al aumentar su tasa en los últimos años (2012) en los más jóvenes; muchos factores tienen una incidencia directa o indirecta en la ideación suicida o en el suicidio propiamente dicho: la violencia intrafamiliar, las condiciones socioeconómicas, el desplazamiento forzado, el matoneo escolar, entre otros, ello se ve reflejado en estudios realizados como el de Díaz, Prados y Ruíz (2004), que evidencia que tanto los agresores como las víctimas de acoso o violencia escolar manifiestan síntomas depresivos, en tanto que en las víctimas se presenta una mayor ideación de muerte [12]. En caso particular de la ciudad de Medellín, a lo anterior se une el accionar de grupos violentos, el micro, el narcotráfico y la falta de oportunidades laborales, lo que en conjunto dimensionan la probabilidad de que conductas suicidas tomen protagonismo.

La situación expuesta anteriormente podría tener efecto en las conductas individuales como la ideación suicida e incluso en llegar a consumar tal idea, esto se da ya que la violencia sea por terceros o intencionales por el mismo sujeto no es innata a los seres humanos, es un aprendizaje, una realidad histórica, humana y socialmente aprendida [11]. Estudios de las desigualdades sociales han puesto de manifiesto que las mujeres, la población de menor nivel socioeconómico o las áreas con mayor privación material presentan unos indicadores de salud que denotan una peor situación ${ }^{13}$. Considerando las causas externas, son las mujeres quienes presentan más afección en su salud debido a las lesiones que ellas se auto infligen, además porque presentan más depresión, utilizan métodos menos letales y más impulsivos, y consultan con mayor frecuencia [14], sin embargo son los hombres quienes suelen ser más contundentes con los medios que utilizan para propiciarse las lesiones.

De acuerdo a los resultados del estudio los hombres fueron protagónicos en el evento considerado, tanto en 2010 como en 2012: por cada 100.000 hombres murieron aproximadamente 9,3 a causa de suicidio, por tanto se les atribuye un $81,4 \%$ de las defunciones, es decir fue la población más vulnerable ante el hecho del suicidio. Lo anterior no dista respecto a los datos mundiales, en cuanto a la tasa de mortalidad por suicidio, es mayor en hombres que en mujeres. La proporción de mortalidad de hombres con respecto a mujeres es de 3:1 en el 2012 [15].

En el estudio carga de la enfermedad 2010 Colombia, mostró que la realidad vivida respecto a la mortalidad por lesiones auto infligidas no distó en gran dimensión de la de Medellín: aparece como la tercera causa de muerte tanto en hombres como en mujeres adolescentes entre 15 y 29 años y la primera entre adultos hombres entre 30 y 44 años [16]. Se destaca en el presente estudio que el grupo de personas adultas también se ven afectadas a causa del suicidio, considerando también que a nivel local se presentó un relevante hecho y fue que en las mujeres de 10 y 14 años se observó un aumento considerable en las tasas de mortalidad (460 por cien mil habitantes) por suicidio, lo que a nivel nacional no se logró visualizar.

En las últimas cuatro décadas del siglo XX, las mayores tasas de suicidio se desplazaron desde los grupos de edades mayores a las personas más jóvenes; Colombia no fue la excepción. Sin embargo, los casos de suicidio en adolescentes allí han permanecido estables en los últimos seis años [14] mientras que en la Ciudad de Medellín es más notoria la fluctuación de ascenso que se ha generado a raíz de la mortalidad por suicidio entre las personas más jóvenes. De acuerdo a la ubicación (comunas y barrios de Medellín) los suicidios se presentaron en su mayoría en la comuna 10 La Candelaria, la comuna 14 El Poblado y la comuna 3 Manrique, aunado a los barrios La Candelaria y Alejandría que presentaron las tasas más elevadas. Valga mencionar que el lugar de ocurrencia del suicidio no coincidió, en la generalidad, con el lugar donde se presentó la muerte, puesto que algunas personas llegaban con vida al centro de atención hospitalaria antes de fallecer. En Medellín de los suicidios consumados, al 18\% se les brindo atención médica.

El estudio revela que la dinámica del evento de interés tuvo un incremento en los casos de mortalidad por suicidio de 2010 al año 2012, afectando al grupo poblacional de jóvenes, quienes representaron una perdida mayor en los años potenciales de vida, situación que genera preocupación pues son en ellos en quienes se concibe el futuro de la sociedad, la mayor productividad del país por su fuerza de trabajo y capacidad intelectual; se sigue presentando un aumento de los casos pese a las diferentes soluciones que en la ciudad se han planteado, por lo que debe prevalecer el desafío de generar alianza y articulación entre políticos, promotores y prestadores de salud para que juntos tomen conciencia de tal problemática y así, surjan estrategias que verdaderamente apunten al cumplimiento de la meta la cual estaría dirigida en pro de disminuir e incluso de evitar al máximo que se presenten casos de mortalidad por suicidio, sin embargo para el alcance de las metas es importante no solo plasmar políticas, sino ejecutarlas adecuadamente unificando fuerzas para realizar acompañamientos individuales y familiares con el fin de mitigar y erradicar los diversos problemas que influyen en gran medida para dar lugar al desencadenamiento de tal evento evitable.

Es de destacar que el estudio presentó una limitante respecto al hecho de ser comparable con otros estudios, ello debido a la metodología utilizada para el cálculo de los APVP, en donde se tomó la esperanza de vida al Nacer de Medellín, la que difiere un poco de la nacional e incluso de otros países.

\section{Agradecimientos.}

A la profesional Sandra Milena Porras Cataño por su invaluable aporte en la construcción de la base de datos.

A la profesora Diana Marcela Marín por su apoyo al inicio del proceso.

A los integrantes del Grupo Investigación de Demografía y Salud por sus contribuciones a la mejoría del producto final

\section{Conflicto de interés}

Los autores manifiestan no tener ningún conflicto de interés 


\section{Referencias}

1. World Health Organization. Preventing suicide: a global imperative. 2014.

2. Guibert Reyes W. Epidemiología de la conducta suicida. Rev Cuba Med Gen Integr [Internet]. 1999, Editorial Ciencias Médicas; [cited 2016 Apr 14];18(2):139-42. Available from: http://scielo.sld.cu/scielo.php?script=sci_arttext\&pid=S086421252002000200007\&lng=es\&nrm=iso\&tlng=es

3. Piña Martínes, Norma Yolanda. El suicidio y sus consecuencias en la familia [Internet]. Asociación mexicana de tanatología, A.C.; 2014. Available from: http://www.tanatologia-amtac.com/ descargas/tesinas/226 el suicidio.pdf.

4. Gómez-Restrepo C, Rodríguez N, C. de Romero L, Pinilla C, López E, Díaz-Granados N, et al. Suicidio y lesiones autoinfligidas, Colombia, 1973-1996. Rev Colomb Psiquiatr [Internet]. 2002;2(2):123-36. Available from: http://www.scielo. unal.edu.co/scielo.php?pid=S0034-74502002000200003\&amp;s cript=sci_arttext\&amp; tlng=es

5. Cardona D, Segura ÁM, Espinosa A, Segura A. Homicidios y suicidios en jóvenes de 15 a 24 años. Colombia, 1998-2008. Biomédica [Internet]. Instituto Nacional de Salud; 2013 May 27 [cited 2016 Apr 14];33(4):574-86. Available from: http:// www.scielo.org.co/scielo.php?script=sci_arttext\&pid=S012041572013000400011\&lng=en\&nrm=iso\&tlng=es.

6. Cendales R, Vanegas C, Fierro M, Córdoba R, Olarte A. Tendencias del suicidio en Colombia, 1985-2002. Rev Panam Salud Pública [Internet]. Organización Panamericana de la Salud; 2007 Oct [cited 2016 Apr 16];22(4):231-8. Available from: http:// www.scielosp.org/scielo.php?script=sci_arttext $\&$ pid $=$ S1020$49892007000900002 \& \operatorname{lng}=$ en\&nrm=iso\&tlng=es

7. Cifuentes Osorio, Sandra Liliana. FORENSIS 2013 3suicidio [Internet]. Colombia; 2013. Available from: http:// www.medicinalegal.gov.co/documents/10180/188820/ FORENSIS+2013+3-+suicidio.pdf/65a683b4-38b2-46a4-b32af2a0884b25bf.

8. Cifuentes Osorio SL. Violencia autonfligida desde el sistema medico-legal colombiano, 2012. [Internet]. Colombia; 2012. Available from: http://www.medicinalegal.gov.co/documents/1 0180/34861/6+5+suicidio+forensis+2012.pdf/654be518-60d04979-b899-f26c83d1bb8c

9. Alcaldía de Medellín. PLAN DE SALUD MUNICIPAL [Internet]. Medellín; 2012. Available from: https://www.medellin.gov. co/irj/go/km/docs/pccdesign/SubportaldelCiudadano_2/ PlandeDesarrollo_0_15/InformacinGeneral/Shared Content/ Documentos/instrumentos/ps/PLAN DE SALUD MUNICIPAL. pdf.

10. Ministerio de Salud. Resolución $\mathrm{N}^{\circ} 008430$ de 1993 [Internet]. 1993. Available from: http://www.unisabana.edu. co/fileadmin/Documentos/Investigacion/comite_de_etica/ Res_8430_1993_-_Salud.pdf.

11. Moreno Clavijo, Gloria María, Posada Posada MI. Años potenciales de vida perdidos por violencia y factores asociados, Valle de Aburrá, 1989- 2009. Universidad de Antioquia; 2013.

12. Palencia Gualdrón B. Manual para la intervención del suicidio en la población escolar de niños y adolescentes. Univ del Rosario [Internet]. Available from: http://repository.urosario. edu.co/bitstream/handle/10336/5027/13923544-2014-. $\mathrm{PDF}$ ? sequence $=2$ \&isAllowed $=\mathrm{y}$

13. Borrell C, García-Calvente M del M, Martí-Boscà JV. La salud pública desde la perspectiva de género y clase social. Gac Sanit [Internet]. SESPAS; [cited 2016 Apr 16];18:02-6. Available from: http://scielo.isciii.es/scielo.php?script=sci_arttext\&pid=S0213$91112004000400002 \& \operatorname{lng}=$ es\&nrm=iso\&tlng=es
14. Rueda-Jaimes GE, Martínez-Villalba AM, Castro-Rueda V a., Camacho P a. Suicidabilidad en adolescentes, una comparación con población adulta. Rev Colomb Psiquiat. 2010;39(4):683-92.

15. Organización Mundial de la Salud, Organización Panamericana de la Salud. Boletín Informativo El Suicidio según vigilancia epidemiológica [Internet]. 2015.

16. Peñaloza Quintero RE, Salamanca Balen N, Rodríguez Hernández JM, Rodríguez García J, Beltrán Villegas AR. Carga de Enfermedad Colombia 2010 [Internet]. Pontificia Universidad Javeriana, editor. Bogotá, D.C.; 2014. Available from: http:// www.javeriana.edu.co/documents/12789/4434885/Carga+de+ Enfermedad+Colombia+2010.pdf/e0dbfe7b-40a2-49cb-848ebd67bf7bc62e 